\title{
El nombre del idioma español según hablantes de Santiago de Chile*
}

\author{
The name of the Spanish language according to Spanish-speaking \\ subjects from Santiago de Chile
}

\author{
Darío Rojas \\ Universidad de Chile, Departamento de Lingüística, Santiago de Chile. \\ Correo electrónico: dariorojasgall@hotmail.com
}

Presentamos resultados parciales del proyecto Linguistic Identity and Attitudes in Spanishspeaking Latin America, que revelan las preferencias de hispanohablantes de Santiago de Chile respecto de la forma de llamar a su propio idioma: español, castellano u otros. Para recoger los datos aplicamos una encuesta directa a 400 sujetos de esta capital estratificados de acuerdo con sexo, edad y grupo socioeconómico. Consideramos que el prestigio normativo asociado a uno de estos nombres, español, es determinante en las respuestas de una parte importante de los encuestados, que declaran usar español a pesar de presuponer que en su habla vernacular lo común es castellano, según sugieren diversas fuentes.

Palabras clave: actitudes lingüísticas, español de Chile, nombre del idioma, español, castellano.

We show partial results of the project Linguistic Identity and Attitudes in Spanish-speaking Latin America, which reveal the preferences of Spanish-speaking subjects from Santiago de Chile regarding the name of their own language: español (Spanish), castellano (Castilian), and others. In order to collect the data we have applied a direct survey to 400 subjects from this city, grouped according to sex, age and socio-economic status. We deem normative prestige as the main factor explaining the answers of an important number of the interviewees. These say that they use mainly español (Spanish), while it is reasonable to assume that they use castellano (Castilian) in their vernacular speech, according to various sources.

Key words: linguistic attitudes, Chilean Spanish, name of the language, español (Spanish), castellano (Castilian).

* Esta investigación se enmarca en el proyecto NFR-193742, Linguistic Identity and Attitudes in Spanish-speaking Latin America - LIAS (Identidad y actitudes lingüísticas en Hispanoamérica - LIAS), del Research Council of Norway (Consejo Noruego de Investigación). 


\section{INTRODUCCIÓN}

Cuando existen distintos nombres para un idioma, como sucede en el caso del español o castellano, sus hablantes inconscientemente tienden a preferir uno de ellos, pero no de forma azarosa, sino que de acuerdo a sus circunstancias socioculturales e históricas atingentes a la lengua y de forma congruente con la ideología lingüística en que se encuentran inmersos. Y si la preferencia se les plantea de forma explícita y consciente, es decir, si se les pregunta directamente cómo llaman o cómo prefieren llamar a su lengua, dichas circunstancias resultan aún más determinantes. Dicho de otro modo, escoger deliberadamente una forma determinada para llamar a la propia forma de hablar puede revelar una actitud lingüística hacia estos nombres y hacia lo que implican acerca de la naturaleza de la lengua a que se refieren.

Una actitud lingüística es una reacción positiva (de aceptación) o negativa (de rechazo) de un individuo o grupo hacia un rasgo lingüístico, hacia sus usuarios o hacia una variedad lingüística completa, motivada por una creencia que usualmente tiene un componente valorativo. Las actitudes, entonces, se componen de tres elementos: un componente cognitivo, es decir, las creencias que les subyacen; uno conativo, esto es, las conductas de rechazo o aceptación observables; y uno afectivo, vale decir, las emociones asociadas a estas conductas (Trudgill y Hernández 2007, s.v. actitudes lingüísticas, cf. también Cargile et al. 1994, Cooper y Fishman 1974, Ladegaard 2000, Liebscher y Dailey-O’Cain 2009, entre otros). Las creencias deben ser entendidas, como han apuntado varios autores (Irvine y Gal 2000; Milroy y Milroy 1999; Schieffelin, Woolard y Kroskrity 1998), como parte de ideologías lingüísticas ${ }^{1}$ o culturas lingüísticas ${ }^{2}$ que se enmarcan a su vez en contextos socioculturales específicos (Coupland y Jawosrki 2004: 37). En cuanto constructos mentales y sociales, culturalmente variables, los sistemas de creencias están sujetos a cambios e intervenciones conscientes o inconscientes.

El conocimiento de las actitudes lingüísticas de los hablantes resulta fundamental para comprender cómo a través del lenguaje se crean y reproducen ideologías e identidades, individuales o grupales, y cómo se forjan y perpetúan prejuicios sociales mediante este instrumento comunicativo. En pocas palabras, el estudio de las actitudes lingüísticas ocupa un lugar central en la comprensión de la relación entre lenguaje y sociedad (Garrett 2001: 630). El potencial de aplicación de sus hallazgos alcanza a algunas de las esferas de mayor relevancia en la vida pública, tales como la educación, la política, la economía y la planificación lingüística. A pesar de la importancia del estudio de las actitudes lingüísticas, en el ámbito hispánico las investigaciones sobre el tema aún son escasas y de alcance parcial (Blas Arroyo 2009). En Chile, las investigaciones relativas al tema (Figueroa 2007, Tassara 1992, Tassara 1993-1994, Valdivieso 1981, Valdivieso 1983) se han enfocado en algún rasgo lingüístico en particular o trabajan con muestras pequeñas. En consecuencia, contamos con un gran

\footnotetext{
"Representations, whether explicit or implicit, that construe the intersection of language and human beings in a social world” (Woolard 1998: 3); “... the ideas with which participants and observers frame their understanding of linguistic varieties and map those understandings onto people, events and activities that are signifiant to them" (Irvine y Gal 2000: 35).

2 “... a relatively abstract and supposedly universal set of beliefs about general concepts such as language, speech, speech community, literacy, etc.” (Del Valle y Gabriel-Stheeman 2002: 10).
} 
vacío de conocimientos respecto de las valoraciones subjetivas respecto del empleo del español tanto a nivel global como a nivel local.

Los resultados finales del proyecto Linguistic Identity and Attitudes in Spanishspeaking Latin America (en adelante, LIAS), de la Universidad de Bergen (Noruega), contribuirán de manera importante a llenar este vacío. En el marco del proyecto LIAS, el primero de alcance global en su tipo, durante los años 2009 y 2010 se han registrado y estudiado las actitudes lingüísticas de hispanohablantes provenientes de 21 ciudades del mundo hispánico, la mayoría correspondientes a capitales. En Santiago de Chile hemos aplicado encuestas a 400 individuos estratificados de acuerdo con estrato socioeconómico (bajo, medio y alto ${ }^{3}$ ), grupo etario (20 a 34, 35 a 54 y 55 o más años) y sexo (hombres y mujeres). La representación de cada uno de los estrato y grupos es proporcional a la que tienen en el universo de la población nacional. Para medir las actitudes lingüísticas de los sujetos se utilizó el método directo (Cargile et al. 1994: 12-13). El instrumento utilizado consiste en un cuestionario de 40 preguntas, de las cuales 26 miden directamente actitudes. Estas elicitan opiniones sobre el español del propio país (incluida su variación interna), sobre el español de otros países (también en comparación con el propio) y sobre el español general. El cuestionario incluye tanto preguntas de respuesta cerrada como preguntas de respuesta abierta.

En el presente artículo presentamos los resultados de la pregunta número 6 del cuestionario: “¿Cómo llama al idioma que habla usted?”. En la sección 2 desarrollamos brevemente los antecedentes relativos al problema del nombre del idioma, y en la sección 3 mostramos y discutimos los hallazgos respectivos que hemos realizado en el marco del proyecto LIAS-Santiago de Chile.

\section{ANTECEDENTES}

Como es bien sabido (Lapesa 1980, Cano 2005), el idioma que hoy se conoce internacionalmente como español es una forma históricamente modificada del latín que se habló en la península ibérica mientras los romanos la dominaron (ss. III a. C. v d. C.) y que se siguió hablando durante los siglos posteriores. El latín hablado, diferente en pronunciación, gramática y vocabulario del latín clásico, adquirió fisonomías particulares (por factores internos y externos) en las diversas provincias del imperio romano (Galia, Hispania, etc.) y, con la formación moderna de unidades políticas, dio origen a los idiomas hoy conocidos como español, portugués, francés, italiano, rumano y otros que integran la familia lingüística romance.

El término romance alude a la condición romana, esto es, 'de Roma (el imperio romano)’, que comparten estas tradiciones históricas del hablar. El adjetivo romance proviene del adverbio latino romanice, que significa 'romanamente, a la manera de los romanos'. Romance, precisamente, convertido ya en un sustantivo, fue el primer nombre que dieron a su forma de hablar los cristianos replegados en las montañas del norte peninsular tras la invasión musulmana del s. viII (Alonso 1979: 10). En la Alta Edad Media los habitantes de la península ibérica aún no reconocían mediante

\footnotetext{
Para la determinación de los estratos socioeconómicos empleamos los datos de ICCOM (Instituto Consultor en Comercialización y Mercado Limitada), en su versión del año 2007 (tablas disponibles en www.iccom.cl). Las equivalencias y proporciones son las siguientes: estrato alto $=\mathrm{ABC} 1$; estrato medio $=\mathrm{C} 2+\mathrm{C} 3$; estrato bajo $=\mathrm{D}+\mathrm{E}$.
} 
distintos nombres las diferencias entre las variedades regionales que conformaban el romance hispánico. Cuando más determinaban el sustantivo romance mediante adjetivos que indicaban una procedencia regional específica: romance leonés, romance castellano, etc., pero castellano y leonés aún no eran nombres de un idioma, así como hoy chileno y argentino no son tales aunque podamos decir que algunos hablan español chileno y otros español argentino. Durante esta misma época, aunque de manera más tardía, los mismos cristianos llamaron también a su forma de hablar lengua vulgar o simplemente vulgar (Alonso 1979: 12-13).

Desde el momento en que el condado de Castilla cobra protagonismo durante la Reconquista (ss. IX-Xv), es decir, durante la recuperación militar de territorios musulmanes por parte de los reinos cristianos del norte peninsular, se produce un vuelco en la historia del nombre de la lengua. Castilla se transformó en un reino, adquirió poder y llegó a absorber a los reinos vecinos (estos se castellanizaron); en consecuencia, su forma de hablar adquirió prestigio hasta llegar a transformarse en la lengua que más tarde identificaría y unificaría a la mayor parte del territorio peninsular. Esta forma de hablar, por haber tenido origen en Castilla, originariamente se denominó romance castellano, luego sustantivado como castellano.

En el tránsito de la Edad Media hacia el Renacimiento y una vez que los Reyes Católicos ya habían logrado unificar gran parte de España, la perspectiva fue distinta. Castellano ya sonaba mucho como "el habla de Castilla", el habla de una región particular dentro de la nueva nación, lo que no concordaba con el hecho de que el idioma al que se refería no se encontraba circunscrito a Castilla, sino que pertenecía a la nación completa. Siendo esta nueva nación conocida con el nombre de España, lo natural era que en adelante se añadiera español a las opciones denominativas. Español es el nombre con que los europeos no españoles de la época conocen esta lengua; español será su nombre internacional y para los mismos españoles se conforma mejor con la perspectiva de grandeza y proyección internacional con que veían sus posesiones culturales. Desde este momento, castellano y español conviven, el primero con un carácter de arcaísmo y el segundo como neologismo propiciado por el momento histórico vivido.

El nombre castellano tiene una especie de revitalización en el s. XVIII, cuando el espíritu cultural posee un tinte más bien erudito e historicista, por lo cual recobra legitimidad llamar a la lengua de acuerdo con su origen territorial. De esta manera, la Real Academia Española titula su primer producto lexicográfico Diccionario de la lengua castellana (también conocido como Diccionario de autoridades, 1726-1739). No obstante, el nombre español consigue imponerse en la gramática y el diccionario académicos desde 1924 y 1925, respectivamente. La preeminencia de español recibió el respaldo de intelectuales de prestigio del ámbito filológico y otros, tales como Ramón Menéndez Pidal y Miguel de Unamuno. Menéndez Pidal, de hecho, ejerció una influencia indirecta en la decisión de la Real Academia Española de cambiar el nombre en los títulos de sus obras (Arenas 2009).

Según la información que ofrece Alonso (1979) para mediados del s. xx, en España castellano era el nombre preferido en zonas rurales, mientras que en la urbe se prefería español. En América, mientras tanto, en la misma fecha existía preferencia general por castellano. Alonso atribuye la situación americana a tradicionalismo arcaizante, pero también a celo patriótico: para las naciones independizadas de España, llamar español a la lengua tiene una connotación indeseada, mientras 
que el nombre castellano resulta menos comprometido al respecto. Por otro lado, "gramáticos y filólogos americanos [...] de hecho han preferido decir castellano, porque su posición ante la lengua es muy análoga a la que tuvo la Academia en el siglo XvIII" (Alonso 1979: 114). El ejemplo más claro de esta actitud es Andrés Bello, quien tituló su principal obra Gramática de la lengua castellana, en la que explicaba que el idioma común de las naciones hispanoamericanas "se llama lengua castellana (y con menos propiedad española)". La preferencia por castellano se refleja en el sistema educativo chileno actual: hasta 1999 la asignatura idiomática escolar se denominaba oficialmente Castellano (hoy se llama Lenguaje y Comunicación) y en la actualidad muchos estudiantes universitarios salen con el título de Profesor de Castellano.

Moreno Fernández y Otero (2007: 34) presentan el siguiente panorama para la fecha presente:

En líneas generales, América del Sur prefiere el término castellano, mientras que Centroamérica y el Caribe se inclinan más por español. En España, la denominación oficial es la de castellano y así se usa también por tradición en diversas áreas, como Castilla o los territorios donde se hablan lenguas co-oficiales (Galicia, País Vasco, Navarra, Cataluña, Valencia, Baleares). Sin embargo el uso de español está también muy extendido, principalmente en el Sur de España. Internacionalmente, el nombre más difundido es español y éste es el preferido por los lingüistas.

Según estos datos, entre la fecha en que Alonso escribe y la actualidad la distribución de ambos nombres ha cambiado: en América castellano coexiste con español (castellano preferido hacia el sur y español hacia el norte), mientras que antes era predominante castellano. En España, por otro lado, al parecer castellano ha abandonado su solar rural y español ha quedado restringido más bien a la zona meridional. El nombre internacional de la lengua, no obstante, sigue siendo español, como ha sido desde el Renacimiento.

Andión (2006) coincide con los autores anteriores en señalar que castellano y español alternan en América, siendo el primero más común en Sudamérica y el segundo en Centroamérica, México y Colombia. La intelectualidad hispanoamericana en general, mientras tanto, prefiere el nombre español. Adicionalmente, esta autora añade datos tomados de los bancos de datos de la Real Academia Española, correspondientes a la búsqueda del sintagma en español/castellano. Si se considera la totalidad de los países de habla hispana, hasta antes de 1971 castellano era más frecuente que español, según muestra el CORDE. En el CREA, en cambio, se aprecia que a partir de 1971 es más frecuente español. Si se considera el detalle por países, España prefería español hasta 1971 y en adelante castellano. Entre los países americanos, usan español los Estados Unidos, Guatemala y Puerto Rico de manera exclusiva, y lo prefieren Cuba, México, Colombia, Argentina y Venezuela. Castellano es el término exclusivo en Bolivia, Paraguay y Perú. En Chile, finalmente, ambos términos muestran una frecuencia similar, aunque con un leve predominio de español (90 casos frente a 86 de castellano).

Por otra parte, las constituciones de una parte importante de los países de Sudamérica, esto es, Colombia, Ecuador, Paraguay, Perú y Venezuela, llaman a la lengua oficial castellano, mientras que la mayoría de las naciones centroamericanas 
(Costa Rica, Cuba, Guatemala, Honduras, Nicaragua y Panamá), lo llaman español. Argentina, Bolivia, Chile, El Salvador, México, República Dominicana y Uruguay omiten mención de lengua oficial en sus constituciones, pero Andión señala que utilizan español en sus documentos oficiales. Con esto, español consigue una preferencia mayoritaria en el discurso oficial de Hispanoamérica.

Desde el punto de vista normativo, la Real Academia Española y la Asociación de Academias de la Lengua Española (2005: s.v. español) consideran aceptables en la lengua ejemplar ambas denominaciones. Sin embargo, recomiendan español "por carecer de ambigüedad” y ser "la denominación que se utiliza internacionalmente”. Señala, asimismo, que resulta preferible reservar castellano para referirse al dialecto hablado antiguamente en el reino medieval de Castilla o en la región española actual del mismo nombre.

\section{EL NOMBRE DE LA LENGUA SEGÚN HISPANOHABLANTES DE SANTIAGO DE CHILE}

¿Qué podemos presuponer, entonces, sobre Chile? Los datos presentados anteriormente sugieren que en Chile el nombre preferido será castellano. La denominación putativamente empleada en este país tendría el carácter conservador y tradicional que le atribuía Alonso (1979) a la opción típica de América y, en especial, por encontrarse en el Cono Sur. También sería congruente con la influencia de los intelectuales de la época de Independencia, que propiciaron el uso de castellano en el ámbito educativo, como por ejemplo Andrés Bello. No obstante, debe tenerse en cuenta que los datos textuales de Andión (2006) muestran que en la lengua escrita chilena se usan ambos términos, con una leve preeminencia de español.

¿Qué revelan los datos que hemos recogido en el proyecto LIAS? La Tabla I muestra los porcentajes en que los encuestados de Santiago de Chile declaran preferir los nombres español, castellano u otros para referirse a su idioma ${ }^{4}$ :

Tabla I. Nombres para la propia lengua preferidos por hispanohablantes de Santiago de Chile

\begin{tabular}{lcc}
\hline Nombre & Respuestas & Porcentaje \\
\hline Español & 202 & $50,5 \%$ \\
Castellano & 191 & $47,8 \%$ \\
Otro nombre & 20 & $5,0 \%$ \\
\hline Total de sujetos & 400 & $100,0 \%$ \\
\hline
\end{tabular}

\footnotetext{
El total de respuestas excede 400, puesto que los encuestados podían responder más de un nombre, por ejemplo “Español o castellano”. En casos como éste, se contabiliza cada nombre por separado, pero la aplicación de SurveyXact calcula los porcentajes sobre un total de 400 sujetos encuestados.
} 
Como se puede ver, la reflexión consciente de los hablantes santiaguinos coincide con los datos de uso del país: español y castellano tienen similar proporción de preferencias, con un ligero predominio del primer nombre.

Debe tenerse en cuenta, sin embargo, que estamos comparando datos de distinto tipo: lo señalado por Andión (2006) corresponde al uso, mientras que nuestros datos muestran qué es lo que los hablantes piensan que usan. No tan sólo eso: el uso que se encuentra registrado en el CREA es principalmente de lengua escrita, probablemente influenciada por la norma y las valoraciones sociolingüísticas respecto de qué es lo correcto. En este mismo sentido, al estar respondiendo a la pregunta de qué es lo que usan, puede que los resultados de LIAS reflejen el deseo de los hispanohablantes de Santiago de Chile de cumplir expectativas respecto de qué es lo más adecuado o prestigioso. ${ }^{5}$ Como señalaba Andión, español tiene preferencia entre los intelectuales hispanoamericanos. Si a esto se añade la decisión de la autoridad lingüística hispánica de favorecer el nombre español, resulta razonable suponer que algunos de los sujetos que declaran usar español en realidad usan ambos términos indistintamente o bien de hecho usan en la oralidad con más frecuencia castellano.

Las variables extralingüísticas consideradas en LIAS, aunque de manera débil, comprueban la putativa influencia del prestigio y la valoración normativa en la elección de nuestros encuestados. En cuanto a estrato socioeconómico, castellano es preferido en los grupos bajo (56,1\% frente a 46,1\% de español) y alto (60\% frente a $40 \%$ de español), mientras que español lo es en el grupo medio (57,2\% frente a 36,7\% de castellano). En lo relativo a la edad de los sujetos, el grupo más joven muestra preferencia por español $(53,8 \%$ frente a $43,4 \%$ de castellano), mientras que el grupo de más edad prefiere castellano (54,3\% frente a 46,8\% de español). El factor sexo, igualmente, muestra influencia en la elección: los hombres prefieren más castellano (50,8\% frente a 46,1\% de español); las mujeres, en cambio, dan preferencia a español $(54,5 \%$ frente a $45 \%$ de español). El perfil variacionista descrito coincide con lo que se conoce sobre las tendencias de ciertos grupos a preferir las variantes prestigiosas (Blas Arroyo 2005: 171, Chambers 2002: 352-355, Wodak y Benke 1998): en nuestro caso, español, la forma de prestigio favorecida por la norma y de alcance internacional, es lo preferido por el estrato medio, por mujeres y por jóvenes. En el caso de estos últimos no puede descartarse la influencia estandarizadora de la escuela, que ha abandonado ya hace poco más de una década el nombre de castellano. Dicho desde el punto de vista de un modelo sociolingüístico distinto, el de mercados lingüísticos (Ash 2002), los grupos en que se acusa la preferencia por español son aquellos que participan más activamente del mercado lingüístico y que, en consecuencia, se ven más influenciados por la norma.

La Tabla II contiene el detalle del 5\% de respuestas que aluden a otros nombres, distintos de español y castellano.

Durante la aplicación de la encuesta, varios de los sujetos daban muestras evidentes de que sentían estar siendo interrogados acerca de cuál era la forma correcta de llamar al idioma, en lugar de lo que planteaba la pregunta directamente, “¿Cómo llama al idioma que habla usted?”. Así, algunos respondían con entonación interrogativa (“¿Español?”), como esperando confirmación por parte del encuestador; otros, tras responder, preguntaban: “¿Así se dice, cierto?”. 
Tabla II. Nombres distintos de español y castellano preferidos por hispanohablantes de Santiago de Chile

\begin{tabular}{lcc}
\hline Nombre & Respuestas & Porcentaje \\
\hline Chileno & 6 & $30 \%$ \\
Español de Chile & 6 & $30 \%$ \\
Español chileno & 2 & $10 \%$ \\
Castellano a lo chileno & 1 & $5 \%$ \\
Castellano chilenense & 1 & $5 \%$ \\
Español a medio hablar & 1 & $5 \%$ \\
Español masticado, champurreado & 1 & $5 \%$ \\
Español medio chancaca & 1 & $5 \%$ \\
Chilenismos & 1 & $5 \%$ \\
\hline Total & 20 & $100,0 \%$ \\
\hline
\end{tabular}

El adjetivo sustantivado chileno, junto con español de Chile, son las etiquetas alternativas preferidas por los encuestados. La mayor parte de las demás denominaciones corresponden a ampliaciones sintagmáticas de español o castellano, mediante las que se especifica un ámbito nacional del idioma ("de Chile", "chileno", "a lo chileno", "chilenense”) o una manera de hablarlo valorada negativamente ("a medio hablar", "masticado, champurreado", "medio chancaca $[=$ de poca prolijidad]"). Las preferencias por otros nombres ocurren sólo en los estratos bajo y medio: el estrato alto utiliza únicamente castellano o español.

\section{CONCLUSIONES}

La elección consciente del nombre de la lengua entre hispanohablantes de Santiago de Chile revela la influencia del prestigio y valoración normativa que cada uno de estos nombres posee en la comunidad panhispánica. La mayoría de las respuestas corresponden a español o castellano. Un poco más de la mitad de los encuestados declara usar español, la forma sancionada normativamente, a pesar de que este modo de denominar al idioma no cuenta con tradición arraigada en el habla local de Chile, cuyos hablantes vernacularmente han preferido llamar castellano a su forma de hablar. La preferencia por español, precisamente, es más acusada en los grupos de mayor sensibilidad o exposición a las formas lingüísticas prestigiosas: estrato medio, jóvenes y mujeres.

Lo dicho nos lleva a concluir a que probablemente nos encontramos ante uno de los numerosos casos en que las creencias, opiniones o declaraciones explícitas de un individuo acerca del lenguaje son incongruentes con su propia conducta y se encuentran influenciadas por las expectativas sociales que intentan satisfacer (Milroy y Milroy 1999: 15-16). Sin embargo, esta hipótesis necesita ser corroborada con el uso actual de los hablantes chilenos tanto en la oralidad como en la escritura. Invitamos a los investigadores a continuar las pesquisas en esta dirección. 


\section{OBRAS CITADAS}

Alonso, A. 1979. Castellano, español, idioma nacional. Historia espiritual de tres nombres. $5 .^{\text {a }}$ edición. Buenos Aires: Losada.

Andión, Ma. A. 2006. “Castellano vs. español: perspectiva panhispánica en la rivalidad terminológica”. Español Actual 85:7-23.

Arenas, J. 2009. "El nombre de la lengua. Lingüística y sociedad a comienzos del siglo XX en España”. Boletín de la Real Academia Española T. LXXXIX, C. CCXCIC. 5-40.

Ash, Sh. 2002. "Social Class". The Handbook of Language Variation and Change. J. K. Chambers, Peter Trudgill y Natallie Schilling-Estes. (eds.). Malden: Blackwell. 402-422.

Blas Arroyo, J. L. 1999. "Las actitudes hacia la variación intradialectal en la sociolingüística hispánica”. Estudios Filológicos 34:47-72.

Blas Arroyo, J. L. 2005. Sociolingüística del español. Desarrollos y perspectivas en el estudio de la lengua española en contexto social. Barcelona: Cátedra.

Cano, R. (coord.). 2005. Historia de la lengua española. 2. ${ }^{\text {a }}$ edición. Barcelona: Ariel.

Cargile, A. C., Giles, H., Ryan, E. B., y Bradac, J. J. 1994. "Language Attitudes as a Social Process: A conceptual model and new directions". Language \& Communication 14,3:211236.

Chambers, J. K. 2002. "Patterns of Variation including Change". The Handbook of Language Variation and Change. J. K. Chambers, Peter Trudgill y Natallie Schilling-Estes. (eds.). Malden: Blackwell. 349-372.

Cooper, R. L. Y Fishman, J. 1974. "The study of language attitudes". International Journal of the Sociology of Language 3:5-20.

Coupland, N. y Jaworski, A. 2004. "Sociolinguistic Perspectives on Metalanguage: Reflexivity, Evaluation and Ideology". Metalanguage: Social and Ideological Perspectives. Nikolas Coupland, Adam Jaworski y Dariusz Galasiński. (eds.). Berlín: Mouton de Gruyter. 15-51.

Del Valle, J. y Gabriel-Stheeman, L. 2002. "Nationalism, hispanismo and monoglossic culture". The Battle over Spanish between 1800 and 2000. Language ideologies and Hispanic intellectuals. José del Valle y Luis Gabriel-Stheeman. (eds.). Londres: Routledge. 1-13.

Figueroa, M. 2007. "Prestigio de las variantes de [tr] en la comuna de Concepción. Estudio sociolingüístico”. Actas del XVII Congreso de Investigación y Enseñanza de la Lingüística. Extraído el 15 de agosto de 2011 desde http://www2.udec.cl/sochil2007/figueroa.pdf

Garrett, P. 2001. "Language Attitudes and Sociolinguistics". Journal of Sociolinguistics 5,4:626-631.

Irvine, J. y GAL, S. 2000. "Language Ideology and Linguistic Differentiation". Regimes of Language: Ideologies, Politics, and Identities. Paul V. Kroskrity. (eds.). Oxford: Oxford University Press. 35-84.

Ladegaard, H. J. 2000. "Language Attitudes and Sociolinguistic Behaviour: Exploring attitudebehaviour relations in language". Journal of Sociolinguistics 4,2:214-233.

Lapesa, R. 1980. Historia de la lengua española. 9. a edición. Madrid: Gredos.

Liebscher, G. y Dailey-O'Cain, J. 2009. "Language Attitudes in Interaction". Journal of Sociolinguistics 13,2:195-222.

Milroy, J. y Milroy, L. 1999. Authority in Language: Investigating Standard English. Londres: Routledge.

Moreno Fernández, F. y Otero, J. 2007. Atlas de la lengua española en el mundo. Barcelona: Ariel.

Real Academia Española y la Asociación de Academias de la Lengua Española. 2005. Diccionario panhispánico de dudas. Madrid: Santillana.

Schieffelin, B., Woolard, K. y Kroskrity, P. (eds.). 1998. Language Ideologies: Practice and Theory. Nueva York: Oxford University Press.

Tassara, G. 1992. “Actitudes lingüísticas ante la variación de /ch/”. RLA 30: 263-271. 
Tassara, G. 1993-1994. "Valoración subjetiva de usos fonéticos alternativos en una muestra porteña”. Nueva Revista del Pacifico 38-39:143-157.

Valdivieso, H. 1981. Valoración subjetiva de los usos lingüísticos. Concepción: Universidad de Concepción.

Valdivieso, H. 1983. "Prestigio y estigmatización: factor determinante en la enseñanza institucionalizada de la lengua materna". RLA 21:137-142.

Wodak, R. y Benke, G. 1998. "Gender as a Sociolinguistic Variable: New Perspectives on Variation Studies". The Handbook of Sociolinguistics. Ed. Florian Coulmas. Malden: Blackwell. 127-150.

Woolard, K. 1998. "Introduction”. Language Ideologies: Practice and Theory. Bambi Schieffelin, Kathryn Woolard y Paul Kroskrity. (eds.). Nueva York: Oxford University Press. 3-47. 